\title{
Variation of Photoneutron Cross Section with Mass Number
}

\author{
N. KARPUZ ${ }^{a *}$, M.C. Boz $^{b}$, B. MAVI ${ }^{b}$ AND I. AKKurT ${ }^{a}$ \\ ${ }^{a}$ Süleyman Demirel University, Physics Department, Isparta, Turkey \\ ${ }^{b}$ Amasya University, Physics Department, Amasya, Turkey
}

\begin{abstract}
Photonuclear reactions play an important role in many different subfields of nuclear astrophysics, and fields, such as nucleosynthesis applications. The photonuclear reaction cross sections, especially the photoneutron reaction cross sections, are the fundamental properties of nuclear systems. In this study, theoretical evaluation of photoneutron reaction cross sections for $27 \leq \mathrm{A} \leq 238$ targets $\left({ }^{27} \mathrm{Al},{ }^{96} \mathrm{Mo},{ }^{181} \mathrm{Ta},{ }^{238} \mathrm{U}\right)$ have been investigated in the incident photon energy range of $7-35 \mathrm{MeV}$. Monte Carlo code TALYS 1.6 was used to calculate the photoneutron cross sections. The results are compared with the earlier experimental reports in literature and the cross section data in the TENDL-2013 nuclear reaction data library, based on subsequent versions of the TALYS nuclear code system. The photoneutron cross section results and the effects of the mass number of target nuclei are discussed.
\end{abstract}

DOI: 10.12693/APhysPolA.128.B-409

PACS: 25.20.-x, 13.85.Lg

\section{Introduction}

A photonuclear reaction is characterized by the photon incident on a target, and one or more products of the reaction being emitted ( $\mathrm{p}, \mathrm{n}, \mathrm{d}, \mathrm{t}, \mathrm{h}, \mathrm{etc}$.$) and de-$ tected [1]. Photonuclear data, describing interactions of photons with atomic nuclei, are of importance for a variety of applications. These applications span from radiation shielding and radiotherapy to inspection technologies and possibly nuclear transmutation [2].

The photonuclear reaction cross sections are the fundamental properties in nuclear systems. The information on total photonuclear cross sections is, in general, good, since these cross sections are obtainable from the photoneutron cross sections, especially for high atomic number (above copper) elements [3, 4].

\section{Models and results}

TALYS is a simulation computer code system for the analysis and prediction of nuclear reactions. The basic objective behind its construction is the simulation of nuclear reactions that involve neutrons, photons, protons, deuterons, tritons, $3 \mathrm{He}$ and alpha particles. TALYS integrates the optical model, direct, preequilibrium, fission and statistical nuclear reaction models in one calculation scheme and gives a prediction for all open reaction channels. The preequilibrium reactions were considered by the two component exciton model. In TALYS, preequilibrium model is the two component exciton model of Kalbach.

Pre-equilibrium nuclear reaction models have been used in calculations. Theoretical cross section calculations are based on the theoretical nuclear reaction models. For this purpose, TALYS $1.6[5]$ two component exciton and TALYS 1.6 preequilibrium models have been

*corresponding author; e-mail: NurdanKarpuz@hotmail.com

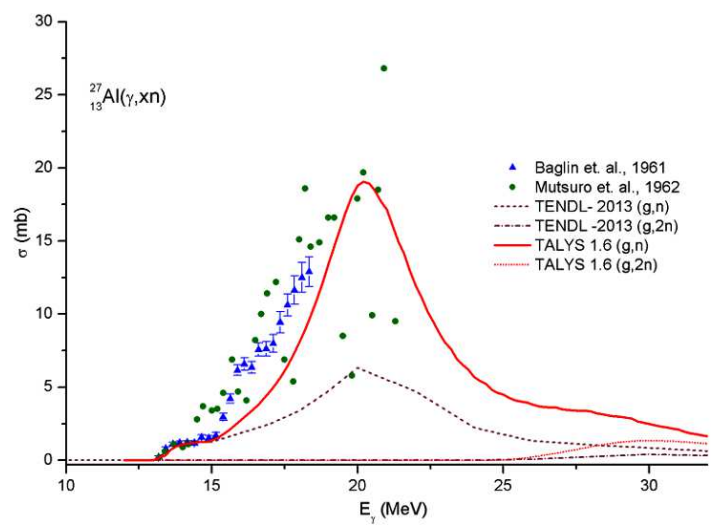

Fig. 1. The cross section for ${ }^{27} \mathrm{Al}(\gamma, x \mathrm{n})$ reaction.

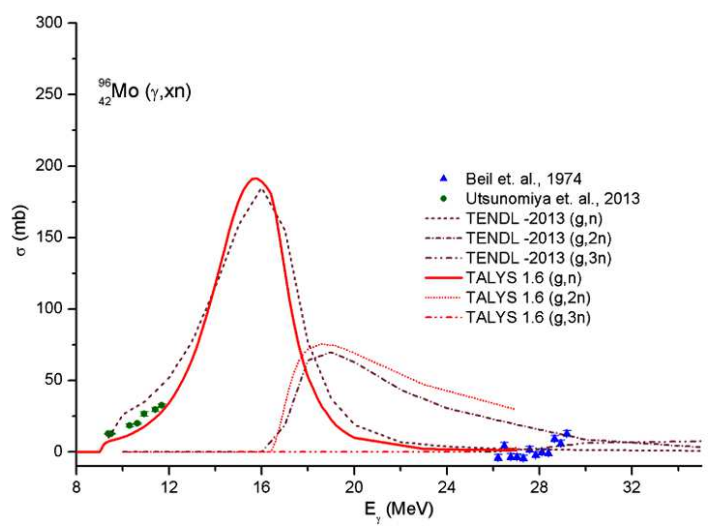

Fig. 2. The cross section for ${ }^{96} \mathrm{Mo}(\gamma, x \mathrm{n})$ reaction.

used to calculate photoneutron cross sections. The calculated $(\gamma, x \mathrm{n})$ reaction cross sections are shown in Figs. 1-4 for $27 \leq \mathrm{A} \leq 238$ targets, respectively. The results have been compared with earlier experimental results from EXFOR [6] databases and cross section data in the TENDL-2013 [7] nuclear reaction data library, based on subsequent versions of the TALYS nuclear code system. 


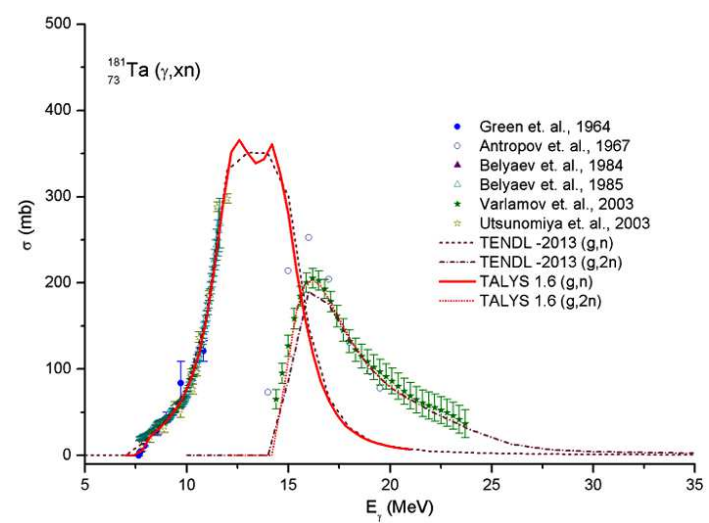

Fig. 3. The cross section for ${ }^{181} \mathrm{Ta}(\gamma, x \mathrm{n})$ reaction.

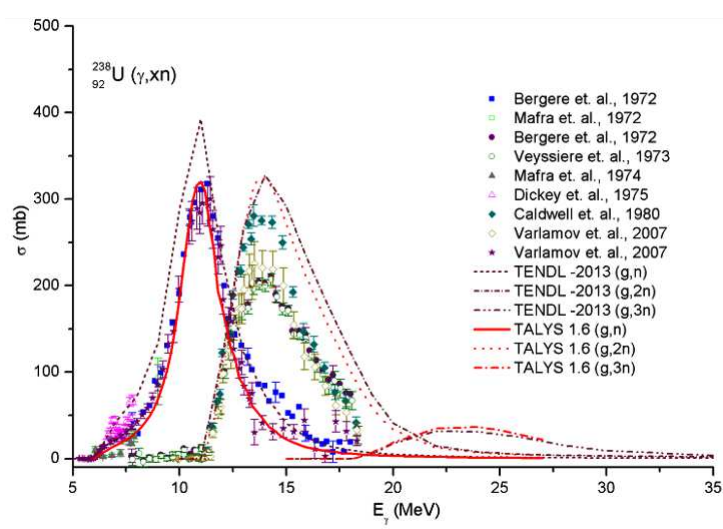

Fig. 4. The cross section for ${ }^{238} \mathrm{U}(\gamma, x \mathrm{n})$ reaction.

\section{Conclusion}

It can be seen from the figures, that the calculated $(\gamma, \mathrm{n})$ reaction cross section results are in good agreement with the experimental data. This is especially true for the calculated $(\gamma, \mathrm{n})$ and $(\gamma, 2 \mathrm{n})$ reaction cross sections for heavy nuclei. TALYS 1.6 two component exciton model is in good agreement with the data and it can be recommended, if the experimental photoneutron cross section data are not available or are unlikely to be produced because of the experimental difficulties.
It can be concluded from this work that the giant dipole resonance effect occurs at photon energies range of about $10-35 \mathrm{MeV}$. This was also shown by other researchers $[8,9]$. It can be concluded from this study, that an increase of photoneutron cross sections has been observed with the increase of mass number. Photoneutron cross section increase is directly proportional to the increase of mass number of nucleus. However the maximum value for $(\gamma, \mathrm{n})$ reaction cross section of target nuclei is inversely correlated with energy.

\section{References}

[1] İ. Akkurt, Chin. J. Phys. 41, 111 (2003).

[2] P. Oblozinsky, Nucl. Sci. Technol. 2, 31 (2002).

[3] J.M. Wyckoff, B. Ziegler, H.W. Koch, R. Uhlig, Phys. Rev. 137, B576 (1965).

[4] İ. Akkurt, N. Karpuz, B. Mavi, N. Demir, Ann. Nucl. Energy 65, 181 (2014).

[5] A. Koning, S. Hilaire, S. Goriely, TALYS-1.6 A Nuclear Reaction Program, User Manual, NRG, The Netherlands, 2013.

[6] EXFOR/CSISRS, Experimental Nuclear Reaction Data File, Brookhaven National Laboratory, National Nuclear Data Center, 2009.

[7] TALYS-based Evaluated Nuclear Data Library, TENDL, 2013.

[8] İ. Akkurt, Ph.D. Thesis, Glasgow University, 1998.

[9] J. Ahrens, Nucl. Phys. A 446, 229 (1985). 